\title{
Service Oriented Architecture (SOA) Design of Electric Power Marketing Information System
}

\author{
Zheyuan WANG ${ }^{1, a}$, Zhou LEI ${ }^{1, b}$, Xiangbo MENG ${ }^{1, c}$, Feng YIN ${ }^{1, d}$ \\ ${ }^{1}$ State Grid Liaoning Electric Power Supply Jinzhou Electric Power Supply Company, China

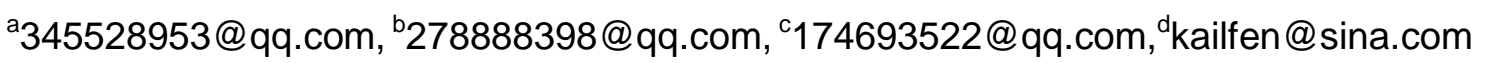

Key words: SOA, ESB, business processes, information interaction

\begin{abstract}
With the change of electric power user's requirements, the business needs in electric power industries changes too, in this case, the new subsystem(SS) or information might be requested to put into. On condition of platform conventions, SOA's application could make different systems comply with Enterprise Service Bus(ESB) information interface integration protocol, so that information interaction and application integration could act among multi tier application system, plug-in application which could solve the traditional end-to-end interface connection problem could be realized in the end.
\end{abstract}

\section{SOA Terms}

SOA is an subassembly model, connecting various different functional units(service) of applications through protocol and interface according to opening standard, its main purpose is to integrate marketing application and reuse subassembly by other system. In way of modeling to describe business relationship, main purpose is to construct those subassembly to the service. Following diagram 1 shows SOA term

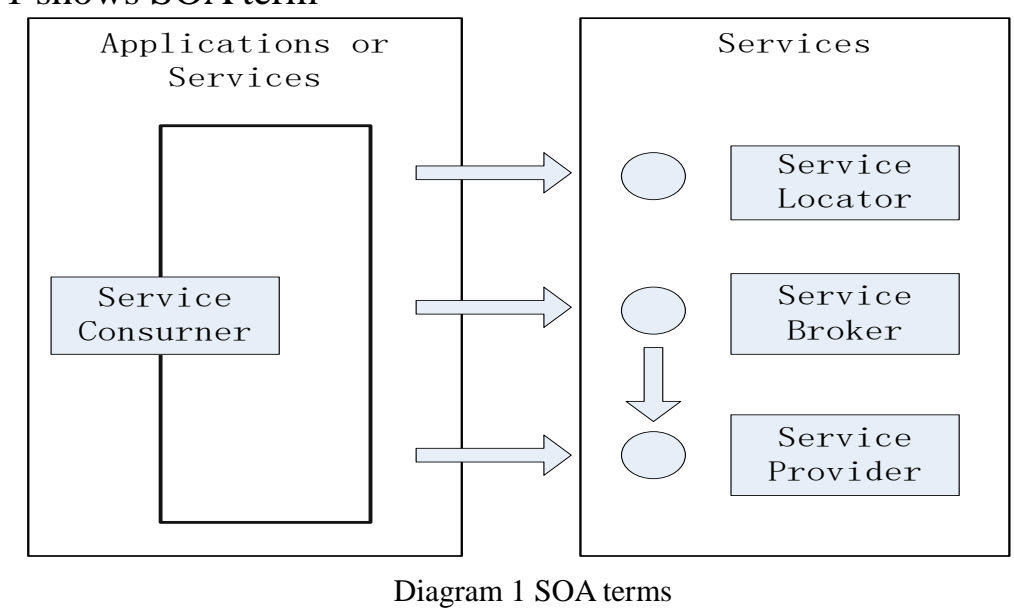

\section{Integration Based on SOA}

As for application system, its service could be deemed as the atom, every read-made applications is one or more service, so that integration would be achieved among them. SOA is the best choice to integrate applications without change any ready-made ones. We have to configure SOA before information interacted among a variety of business information systems, and ESB is applied on this condition. ESB could extract each business subsystem and publish them publicly, at the same time, open calling method and function of all subassembly, so that information exchange among a variety of subsystems could call what information(function) should be exchanged according to agreed method. This will not only eliminate the complex coupling relationship among each subsystems, but also make them get relative independence, security and integrity. ESB is a core hub for data interaction and business integration among applications. ESB logic framework see to following 
diagram 2

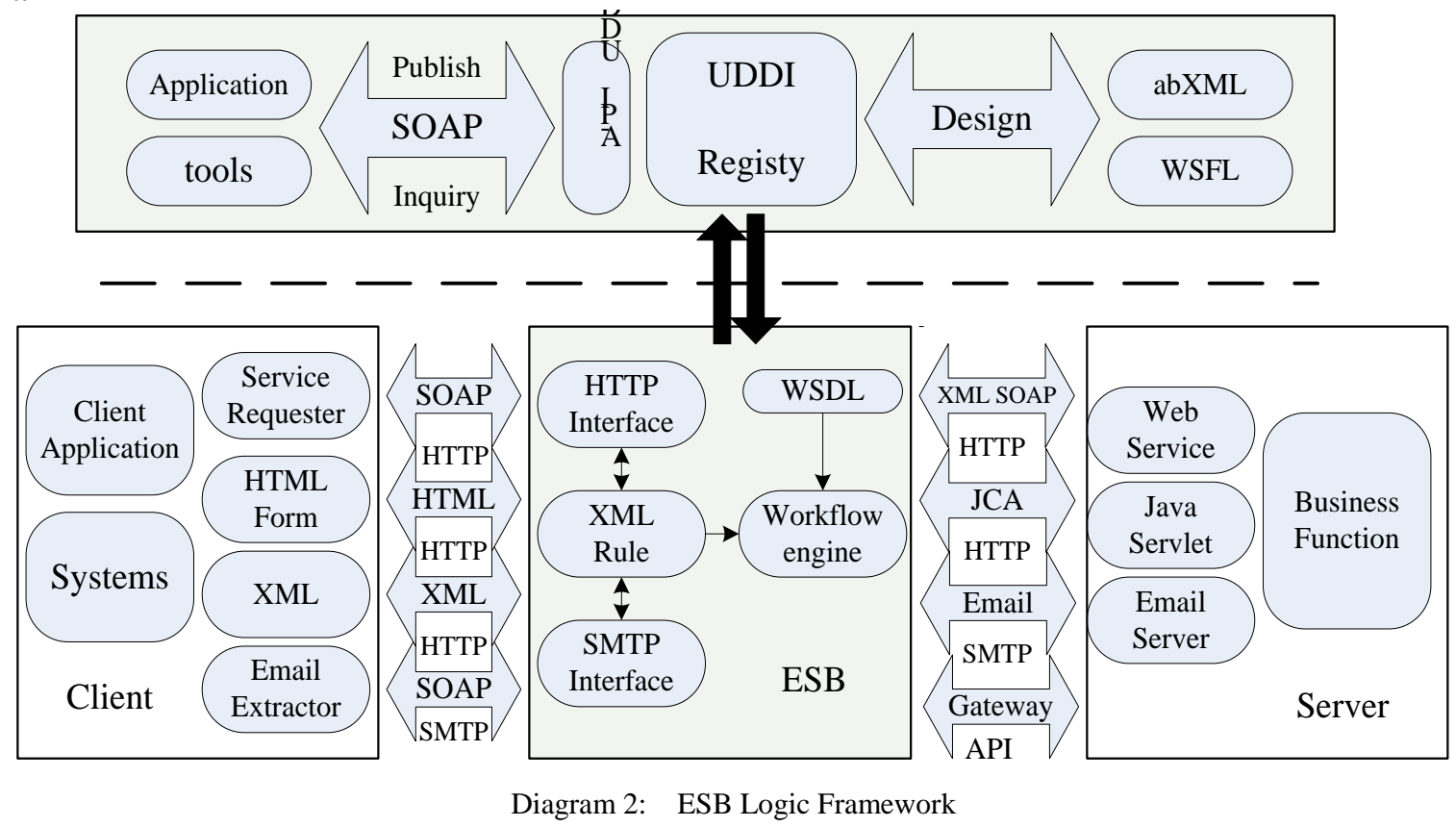

ESB is a core hub for data interaction and business integration among various applications systems, information transfer among all service could be controlled and achieved via a variety of information transferring protocol, so that end-to-end business process mode among all interactive information could be achieved finally.

\section{Business Process Definition}

Business process definition is a service processing procedure which is caused by application service according to business requirements, it not only define relevant management of business process, but also support task allocation, multi branch, parallel and user right administration. Firstly encapsulate the custom-made work flow, then package it into a .NET assembly, in order that it could be convenient to be called and integrated by platform and other business. Because integration rules are stored in XML format, it will never reply on a certain specific middleware process components.

\section{Process driven engine}

Process driven engine is realized by application of middleware integrated components.

\section{Service Publishing and Searching Platform}

Service publishing and searching platform apply universal description, discovery and integration(UDDI) service, which could meet essential information interactive integration requirement in information integration environments and support various Web service publishing, query, maintenance on the platform, so the application integration based on Web service is realized.

\section{Platform Basic Service}

Platform Basic Service, mainly include: Route control, Security certification, Transmission encryption, Application gateway, XML parsing, Service discovery, Log tracking, Copy processing

\section{Platform Rule File Base}

Both regular file base and configuration file on platform are accessed in XML format, mainly include: application integration rule base, date interaction rule base, standard error information database, WSDL service describing base.

\section{Service Oriented Architecture (SOA)Design}

Following diagram 3 is is a graph of information interactive architecture between electric power marketing management system and subsystem on ESB platform, full information interaction process is represented in this graph. 


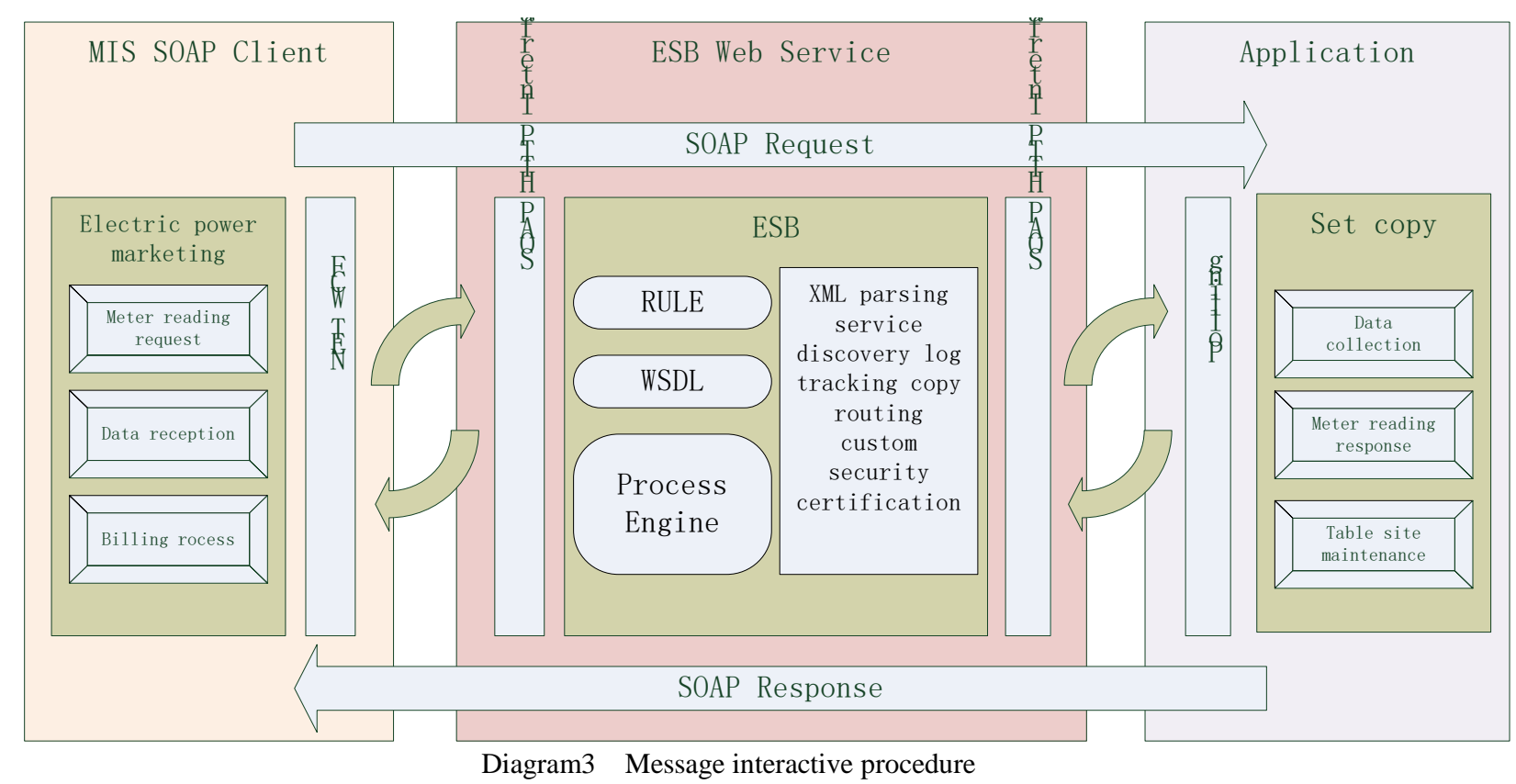

\section{Information Interaction Specification}

Standard SOAP protocol is adopted when systems are interacting, SOAP underlying transport protocol actually, for example, HTTP and SMTP are connected through binding same message frame. In simple terms, SOAP is a open standard protocol of XML+ RPCover HTTP, making communication interaction between Web service provider and requester not blocked by firewall. SOAP is a cross platform protocol, every remote call through network could be encapsulated by it. XML message is transmitted through HTTP protocol, although other parse files are requested during this transmission, both HTTP and XML are open standard specification, furthermore, HTTP is a transmission protocol which is widely used in Web and could pass through the firewall, because all of these, SOAP is being widely used.

\section{Service Specification for Interaction Interface}

(1) Interface type of service

The interface of ESB platform can be divided into client(that's service requester) interface and server (that's service provider) interface. For the client side, ESB platform could be deemed as a Web service, who could provide SOAP protocol visit of MTP to them. SOAP message could be bound with different underlying protocol, meanwhile, among various message transmission it can make SOAP client provide classic library, COM object .etc. For server side, platform adopt SOAP Over HTTP/SMTP to realize function request and information interaction.

(2) Access to service requester

From the view of requester, ESB is not only a Web service provider, but also a Web Service Proxy. When act as a Web service provider, ESB would publish some methods, responding to all kinds of requests from requesters. And when act as a Web Service Proxy, it will provide a UDDI platform, on which other service provider would register and publish service (like Web service, APIs .etc) what would be audit by ESB first, and be called by clients after final integration and optimization.

(3) Access to service provider

From the view of provider, ESM is just a request part. A service provider doesn't care about the first of the request, but only take action to SOAP encapsulation request, and return processing result to ESB directly.

\subsection{SOAP Format}




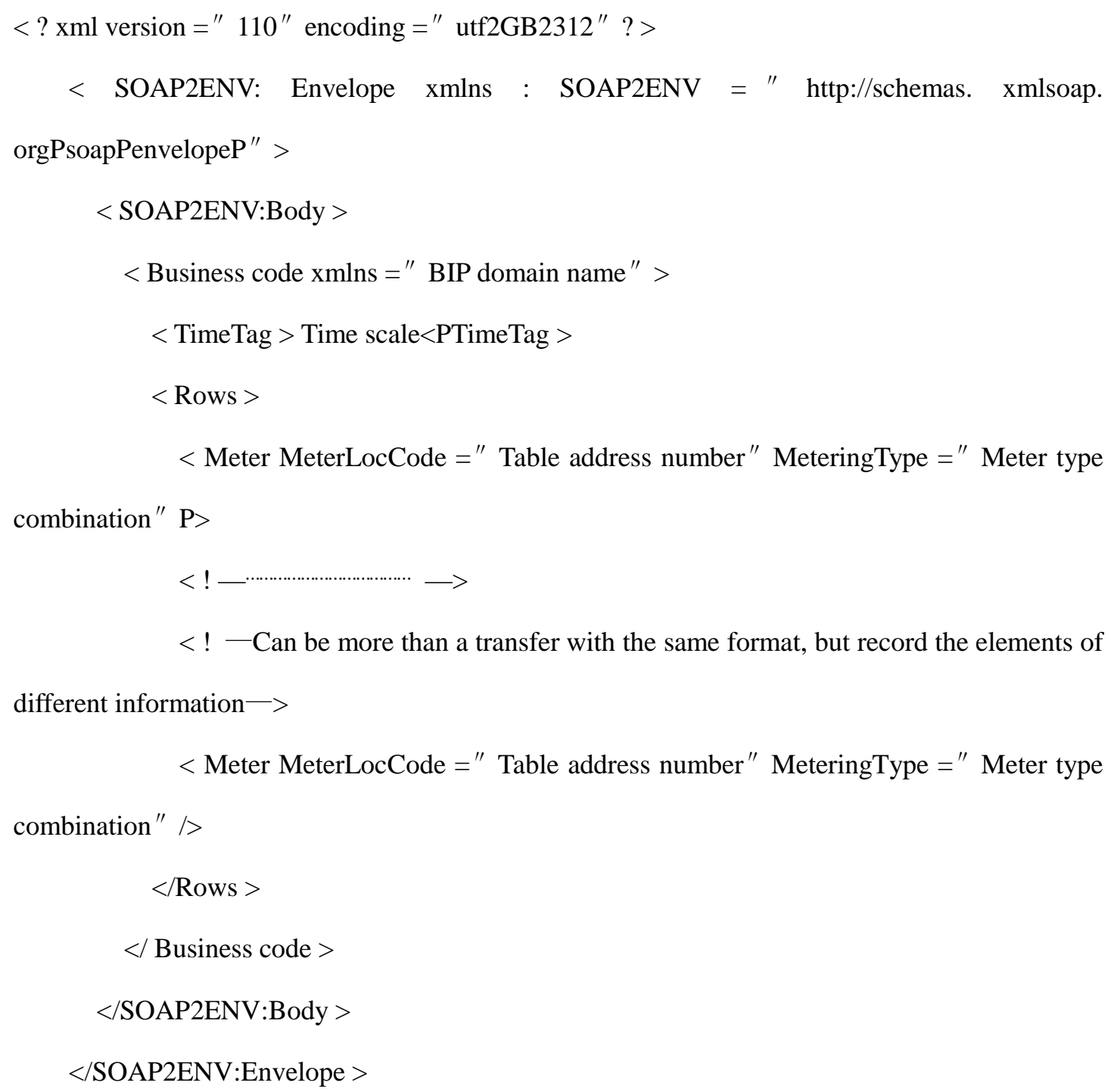

\section{Conclusion}

In this paper, a variety of subsystem information exchanges are achieved based on SOA(Service of Architecture), B/S (Browser/Server) system and application of Enterprise Service Bus (ESB). The design is mainly based on the thinking of "customer centered", Timely, Completely, Accurately and Reliably collect, store and transmit related information, in order to provide accurate ,fast information query service to related departments and electric power users. SOA design and specific function are realized in some degree.

\section{Reference}

[1] Booch G.2005.Object-oriented analysis and design with applications. Addison Wesley Professional

[2] David Geer.2003.Taking steps to secure Web services[J].Computer,36(10):14-16

[3] David B.Ingham,Santosh K.Shrivastava.2000.Constructing dependable Web services[J].Internet Computing,IEEE,4(1):25-33

[4] Eric Newcomer,Greg Lomow.2004.Understanding SOA with Web Services[M].The front page.America:Addison-Wesley Professional

[5] Francisco Curbera,Matthew Duftler ect.2002.Unraveling the Web services web:an introduction 
to SOAP,WSDL,and UDDI[J].Internet Computing,IEEE, 6(2):86-93

[6] John Viega,Jeremy Epstein.2006. Why applying standards to Web services enough[J].Security\&Privacy Magazine ,IEEE,4(4):25-31

[7] Panagiotis Louridas.2006.SOAP and Web Services[J].Software,IEEE, 23(6):62-67 